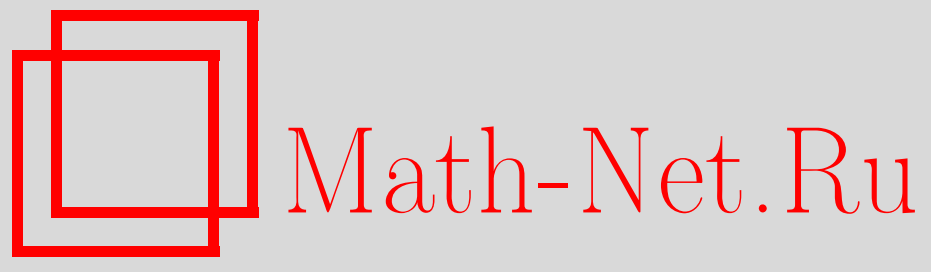

Д. Н. Иванов, Однородные коммутативные ортогональные разложения полупростых алгебр, УМН, 2007, том 62, выпуск 6, 173-174

DOI: https://doi.org/10.4213/rm8531

Использование Общероссийского математического портала Math-Net.Ru подразумевает, что вы прочитали и согласны с пользовательским соглашением http://www . mathnet.ru/rus/agreement

Параметры загрузки:

IP: 3.91 .87 .62

26 апреля 2023 г., 08:12:51

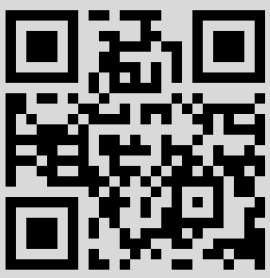




\title{
Однородные коммутативные ортогональные разложения полупростых алгебр
}

\author{
Д. Н. Иванов
}

В статье доказывается теорема о строении алгебр, допускающих ортогональное разложение (OP) в сумму полупростых $d$-мерных коммутативных подалгебр. Доказанная теорема - важный шаг на пути исследования гипотезы о делимости размерности таких алгебр на $d^{2}$. В [1] эта гипотеза была доказана в случае $d=2$. Также, формулируется гипотеза, обобщающая старую гипотезу о порядках конечных плоскостей и сравнительно недавние гипотезы об аффинных 2-схемах и простых алгебрах Ли типа $A_{n}$, допускающих ортогональные разложения в сумму картановских подалгебр.

Напомним основные определения. Пусть $\mathbf{A}$ - ассоциативная конечномерная над полем комплексных чисел $\mathbb{C}$ алгебра, через $\operatorname{Tr}_{\mathbf{A}}$ обозначим след регулярного представления $\mathbf{A}$. Тогда $\operatorname{Tr}_{\mathbf{A}} x y$ является симметричной инвариантной формой на $\mathbf{A}$, которую мы будем называть формой следа. Известно, что алгебра $\mathbf{A}$ полупроста тогда и только тогда, когда форма следа невырождена на А. Далее будут рассматриваться полупростые алгебры и термин "алгебра" будет обозначать ассоциативную конечномерную над $\mathbb{C}$ полупростую алгебру. Таким образом, по теореме Веддербарна алгебра $\mathbf{A}$ изоморфна конечной прямой сумме матричных алгебр $\mathrm{M}_{n}(\mathbb{C})=\mathrm{M}_{n}$.

ОПРеДЕЛЕниЕ 1. Семейство неединичных собственных подалгебр $\mathscr{D}=\left\{\mathbf{B}_{i}, i=\right.$ $1, \ldots, r\}$ образует ортогональное разложсение (OP) алгебры $\mathbf{A}$, если все подалгебры $\mathbf{B}_{i}$ полупросты, содержат единичный элемент $\mathbf{1}_{\mathbf{A}}$ алгебры $\mathbf{A}$ и выполняется условие ортогональности: алгебра $\mathbf{A}$ является прямой суммой попарно ортогональных подпространств $\mathbf{A}=\left\langle\mathbf{1}_{\mathbf{A}}\right\rangle \oplus \mathbf{B}_{1}^{\circ} \oplus \cdots \oplus \mathbf{B}_{r}^{\circ}$, где $\mathbf{B}_{i}^{\circ}=\left\{x \in \mathbf{B}_{i} \mid \operatorname{Tr}_{\mathbf{A}} x=0\right\}$.

Если все подалгебры ОР $\mathscr{D}$ коммутативны, то $\mathscr{D}$ называется коммутативным. Если все подалгебры в $\mathscr{D}$ изоморфны алгебре $\mathbf{B}$, то $\mathscr{D}$ называется однородным OP mu$n a \mathbf{B}$ (или просто OP типа $\mathbf{B}$ ). Через $k \mathrm{M}_{n}$ будем обозначать прямую сумму $k$ экземпляров алгебры матриц $\mathrm{M}_{n}$. Однородное $\mathrm{OP}$ типа $d \mathrm{M}_{1}$ называется $d$-разложением.

Отметим, что понятие однородного коммутативного ОР обобщает ряд известных понятий. Так, матрицы Адамара порядка $n$ эквивалентны 2-разложениям алгебры $n \mathrm{M}_{1}$, конечные аффинные плоскости порядка $n$ эквивалентны $n$-разложениям алгебры $n^{2} \mathrm{M}_{1}$, аффинные 2-схемы эквивалентны однородным ОР коммутативных алгебр, ортогональные разложения простых алгебр Ли типа $A_{n-1}$ эквивалентны $n$-разложениям алгебры $\mathrm{M}_{n}$. В [2] доказана следующая фундаментальная теорема.

Теорема 1. Алгебра А является свободным (левым, правым) модулем над каждой подалгеброй из семейства, образующего коммутативное ОР алгебры $\mathbf{A}$.

Применяя теорему 1 к $d$-разложениям, получаем следующую лемму.

ЛЕмма 1. Пусть подалгебры $\left\{\mathbf{B}_{1}, \ldots, \mathbf{B}_{r}\right\}$ образуют d-разложение алгебры $\mathbf{A}$, $\operatorname{dim} \mathbf{A}=n$. Пусть $\operatorname{Pr}\left(\mathbf{B}_{s}\right)=\left\{e_{1 s}, \ldots, e_{d s}\right\}-$ примитивные идемпотенты подалгеб$p \iota \mathbf{B}_{s}, s=1, \ldots, r$. Тогда

1) $\operatorname{Tr}_{\mathbf{A}} e_{1 s}=\cdots=\operatorname{Tr}_{\mathbf{A}} e_{d s}=n / d$ для любого $s=1, \ldots, r$, в частности, $n$ кратно $d$;

2) элементы $\mathbf{1}_{\mathbf{A}}, e_{j s}, j=1, \ldots, d-1, s=1, \ldots, r$, образуют базис в $\mathbf{A}$, в частно$c m u, r=(n-1) /(d-1)$;

3) для любого $X \in \mathbf{A}$

$$
n \operatorname{Tr}_{\mathbf{A}} X^{2}=d \sum_{s=1}^{r}\left(\left(\operatorname{Tr}_{\mathbf{A}} X e_{1 s}\right)^{2}+\cdots+\left(\operatorname{Tr}_{\mathbf{A}} X e_{d s}\right)^{2}\right)-(r-1)\left(\operatorname{Tr}_{\mathbf{A}} X\right)^{2} .
$$

Следующая теорема является основной.

Работа выполнена при поддержке РФФИ (грант № 06-01-00485-а). 
Теорема 2. Если алгебра $\mathbf{A}=v \mathrm{M}_{1} \oplus \mathrm{M}_{n_{1}} \oplus \cdots \oplus \mathrm{M}_{n_{k}}$, где $n_{i}>1$ nрu $i=1, \ldots, k$, допускает d-разложение, содержащее $r$ подалгебр, то

1) $n_{1}, \ldots, n_{k}$ кратны $d$,

2) $v$ кратно d и существует разрешимая блок-схема на $v$ точках, содержащая $r$ классов параллельных блоков, так что каждые две точки встречаются вместе в $(r-1) / d$ блоках и мощности блоков сравнимы с $v / d$ по модулю $d$.

ДокАЗАТЕЛЬСтво. Пусть $\mathrm{tr}$ - след матрицы, $E_{m}$ - единичная матрица порядка $m, X=\left(X_{0}, X_{1}, \ldots, X_{k}\right) \in \mathbf{A}$, где $X_{0}=\left(x_{1}, \ldots, x_{v}\right) \in v \mathrm{M}_{1}, X_{i} \in \mathrm{M}_{n_{i}}, i=1, \ldots, k$. Тогда $\operatorname{Tr}_{\mathbf{A}} X=x_{1}+\cdots+x_{v}+n_{1} \operatorname{tr} X_{1}+\cdots+n_{k} \operatorname{tr} X_{k}$. Пусть подалгебры $\left\{\mathbf{B}_{1}, \ldots, \mathbf{B}_{r}\right\}$ образуют $d$-разложение алгебры $\mathbf{A}, \operatorname{Pr}\left(\mathbf{B}_{s}\right)=\left\{e_{1 s}, \ldots, e_{d s}\right\}$. Будем отождествлять элементы алгебр $v \mathrm{M}_{1}, \mathrm{M}_{n_{1}}, \ldots, \mathrm{M}_{n_{k}}$ с их каноническими образами в алгебре А. Положим $\operatorname{dim} \mathbf{A}=n$, тогда по лемме 1 имеем $r=(n-1) /(d-1)$.

1) Покажем, что $n_{1}, \ldots, n_{k}$ кратны $d$. Подставив в (1) $X=\left(0, \ldots, 0, E_{n_{i}}, 0, \ldots, 0\right)$, где $E_{n_{i}}$ - единичная матрица в $\mathrm{M}_{n_{i}}$, получим

$$
n n_{i}^{2}=d \sum_{s=1}^{r} n_{i}^{2}\left(\left(\operatorname{tr} E_{n_{i}} e_{1 s}\right)^{2}+\cdots+\left(\operatorname{tr} E_{n_{i}} e_{d s}\right)^{2}\right)-(r-1) n_{i}^{4} .
$$

Положим $\operatorname{tr} E_{n_{i}} e_{j s}=a_{j s}$. Тогда $a_{1 s}+\cdots+a_{d s}=n_{i}$, и так как $E_{n_{i}} e_{j s}$ является идемпотентом в $\mathrm{M}_{n_{i}}$, то $a_{j s} \in \mathbb{Z}$. В этом случае несложно показать, что либо $a_{1 s}^{2}+\cdots+a_{d s}^{2} \geqslant n_{i}^{2} / d+(d-1) / d$, либо $a_{1 s}=\cdots=a_{d s}=n_{i} / d$. Пусть не выполняется последнее условие. Тогда, после сокращения (2) на $n_{i}^{2}$, выводим

$$
n=d \sum_{s=1}^{r}\left(a_{1 s}^{2}+\cdots+a_{d s}^{2}\right)-(r-1) n_{i}^{2} \geqslant d r\left(\frac{n_{i}^{2}}{d}+\frac{d-1}{d}\right)-(r-1) n_{i}^{2}=n+n_{i}^{2}-1 .
$$

Это противоречит тому, что $n_{i}>1$. Значит, $a_{1 s}=\cdots=a_{d s}=n_{i} / d \in \mathbb{Z}$.

2) В силу леммы $1 n$ кратно $d$, и в силу пункта 1) $n_{i}$ кратны $d$, значит, $v=$ $n-n_{1}^{2}-\cdots-n_{k}^{2}$ кратно $d$. Обозначим через $\left(b_{j s}^{1}, \ldots, b_{j s}^{v}\right)$ каноническую проекцию идемпотента $e_{j s}$ на подалгебру $v M_{1}$. Подставим в $(1) X=\left(\left(x_{1}, \ldots, x_{v}\right), 0, \ldots, 0\right)$ :

$$
n\left(x_{1}^{2}+\cdots+x_{v}^{2}\right)=d \sum_{s=1}^{r} \sum_{j=1}^{d}\left(x_{1} b_{j s}^{1}+\cdots+x_{v} b_{j s}^{v}\right)^{2}-(r-1)\left(x_{1}+\cdots+x_{v}\right)^{2} .
$$

Через $N$ обозначим $(v \times r d)$-матрицу, составленную из столбцов $\left(b_{j s}^{1}, \ldots, b_{j s}^{v}\right)^{t}$. Из (3) получаем $d N N^{t}=n E_{v}+(r-1) J_{v}$, где $J_{v}$ - матрица из одних единиц. Следовательно, $N$ является матрицей инцидентности искомой блок-схемы. В силу п. 1) мощности блоков сравнимы с $n / d$, а значит, и с $v / d$ по модулю $d$. Теорема 2 доказана.

ЗАмечАния. 1) Если алгебра $\mathbf{A}$ коммутативна, т. е. $v=\operatorname{dim} \mathbf{A}$, то $\operatorname{dim} \mathbf{A}$ делится на $d^{2}$, так как (в обозначениях теоремы 2) $e_{i s_{1}} e_{j s_{2}}$ есть идемпотент и, значит, при $s_{1} \neq s_{2} \operatorname{dim} \mathbf{A} / d^{2}=\operatorname{Tr}_{\mathbf{A}} e_{i s_{1}} e_{j s_{2}} \in \mathbb{Z}$.

2) Для некоторых значений $d \neq 2$ существуют разрешимые блок-схемы с указанными в теореме 2 свойствами, для которых $v$ не делится на $d^{2}$.

ГипотезА 1. Если алгебра $\mathbf{A}$ допускает $d$-разложение, $\operatorname{mo} d=p^{m}$ для некоторого простого р и натурального $n$. При этом если $d \neq 2$, то $\operatorname{dim} \mathbf{A}$ является степенъю $d$.

Автор благодарен М. В. Зайцеву и А. В. Михалёву за внимание к работе.

\section{Список литературы}

[1] Д. Н. Иванов, УМН, 60:2 (2005), 163-164; англ. пер.: D. N. Ivanov, Russian Math. Surveys, 60:2 (2005), 357-358. [2] Д.Н. Иванов, Матем. сб., 189:12 (1998), 83-102; англ. пер.: D. N. Ivanov, Sb. Math., 189:12 (1998), 1819-1838.

Д. Н. Иванов (D. N. Ivanov)

Тверской государственный университет E-mail: dni@tvcom.ru
Представлено А. В. Михалёвым Принято редколлегией 29.09.2007 